Artículo de Educación 



\title{
Usos y realidades de las tecnologías de la información y la comunicación en las instituciones educativas del sector oficial del Municipio de Sincelejo' ${ }^{1}$
}

\author{
Uses and Realities of Information and Communication Technologies in Public \\ Schools of Sincelejo
}

\author{
Claudia Cristina Lengua Cantero² \\ Laydyt María Lambraño Pérez ${ }^{3}$
}

\begin{abstract}
Resumen
El presente artículo analiza el nivel de apropiación y uso de las tecnologías de la información y la comunicación (TIC) por parte de los estudiantes, docentes y directivos de las instituciones educativas del sector oficial del municipio de Sincelejo, caracterizando para ello la infraestructura tecnológica, los usos que le da la comunidad académica, los conocimientos acerca de las Tecnologías de la Información y la Comunicación, TIC y su implementación en el currículo gracias a la gestión de las directivas. Se utilizó un enfoque mixto, con el propósito de describir e interpretar la realidad observada, su naturaleza fenomenológica a partir de tres categorías de análisis, conocimientos y usos, infraestructura tecnológica, y gestión tecnológica. La población estudio cursa los grados 10 y 11 de las instituciones educativas. Para la obtención de datos se aplicó un cuestionario a docentes y estudiantes y una entrevista a directivos. De acuerdo con lo anterior, los vectores causa-efecto acerca de las TIC no radican exclusivamente en la existencia de equipos, salas acondicionadas, jornadas de capacitación a los docentes; el problema tiene fuentes en la cultura organizacional: los nuevos ambientes de aprendizaje suponen retos a quienes los operan y, a su vez, nuevas estructuras y herramientas para responder a nuevos propósitos y filosofías curriculares.
\end{abstract}

Palabras clave: tecnología de la información, medios, enseñanza, tecnología.

\begin{abstract}
This paper analyzes the level of ownership and use of Information Technology and Communication by students, teachers and administrators of educational institutions in the formal sector of the municipality of Sincelejo, characterizing the technological infrastructure for it, uses that gives the academic community, knowledge about Information Technology and Communication and it inclusion in the curricula, by the management of the directives. A mixed approach was used in order to describe and interpret the observed reality, its phenomenological nature from three categories of analysis, knowledge and practices, technological infrastructure, and technology management. The population of study is in
\end{abstract}

1 Esta investigación hace parte del proyecto "Uso y apropiación de las tecnologías de la información y la comunicación en las instituciones educativas del sector oficial del municipio de Sincelejo", investigación institucionalizada financiada por la Corporación Universitaria del Caribe (Cecar).

2 Magíster en Gestión de la Tecnología Educativa, docente de la Corporación Universitaria del Caribe, investigadora del Grupo Idead avalado por Colciencias Categoría C. E-mail: claudia.lengua@cecar.edu.co.

3 Especialista en Ingeniería de Software, docente de la Corporación Universitaria del Caribe, investigadora del Grupo Idead avalado por Colciencias Categoría C. E-mail: laudyt.lambrano@cecar.edu.co 
grades 10 and 11 of the educational institutions. We apply a questionnaire to teachers and students for data collection, and an interview with management. According to the above, the cause-effect vector about Information Technology and Communication are not solely on the existence of equipment, upgraded rooms, training sessions for teachers; the problem has sources in the organizational culture, new learning environments present challenges to those who operate and in turn new structures and tools to respond to new curricular aims and philosophies.

Keywords: information technology, media, education, technology.

Recibido: 13 de julio de 2013

Aceptado: 17 de septiembre de 2013

En los últimos años, el uso de las Tecnologías de la Información y la Comunicación (TIC) se ha ampliado notablemente, facilitándole a muchas personas el acceso al conocimiento y achicando la llamada brecha digital. Este contexto hace que la escuela, como nunca antes, haya perdido la hegemonía respecto a ser el espacio por excelencia en el que se concentraba el saber, abriéndose a nuevos ambientes de aprendizaje más interactivos e incluyentes. Relativo a ello, la Unesco (2002: 28) plantea lo siguiente:

Los docentes y estudiantes necesitan estar preparados para empoderar a los estudiantes con las ventajas que les aportan las TIC. Escuelas y aulas -ya sean presenciales o virtualesdeben contar con docentes que posean las competencias y los recursos necesarios en materia de TIC y que puedan enseñary aprender de manera eficaz las asignaturas exigidas, integrando al mismo tiempo en su enseñanza conceptos y habilidades de estas.

Con esto se da por sentado que docentes y estudiantes integran un proceso de enseñanzaaprendizaje mediado y dinamizado por las TIC, en el cual el aula, la institución y la sociedad conforman nuevos ambientes educativos amplios e incluyentes.

No obstante lo planteado por la Unesco, el panorama que se observa en las instituciones educativas del sector público del municipio de Sincelejo (Colombia), es distinto: los procesos de formación mediados por TIC son incipientes; a la precaria dotación tecnológica y las condiciones locativas inadecuadas se suma el bajo nivel de conocimiento, aplicación e incorporación de las TIC por parte de los docentes y estudiantes, quienes, en términos, generales se rezagan frente a las inquietudes y ritmos de los currículos, subutilizando en alto porcentaje los equipos y recursos tecnológicos disponibles en las instituciones educativas del sector.

De esta forma, la preocupación global por incorporar las TIC de forma efectiva al proceso de formación de los jóvenes es recurrente. En Chile el proyecto Enlaces, del Ministerio de Educación Chileno, ha logrado en los últimos años que 6.500 establecimientos cuenten con planteles de uso educativo de las TIC, que 22.000 docentes sean capacitados por competencias en uso de tecnologías e integración curricular, que se implementen 14 modelos de integración curricular, que se visibilicen 300 experiencias pedagogías el uso de TIC, que cerca de 1.500 establecimiento con software educativos estén financiados por el fondo ministerial, que 3.500 establecimientos cuenten con subsidio para la conectividad, que más de 1.500 establecimientos municipales tengan laboratorios móviles y que 7.000 salas de clases cuenten con tecnología de puna (data telon, notebook y recursos digitales). Además, este proyecto ha contribuido a mejorar la calidad de la educación mediante la informática educativa y el desarrollo de una cultura digital en la ciudadanía, con calidad, equidad y pertinencia (Ministerio de Educación Nacional, Gobierno de Chile, 2011). 
Esta misma preocupación la comparte Colombia, donde el Ministerio de Educación Nacional ha venido aunando esfuerzos para adelantar procesos que faciliten el acceso a las TIC por parte de la comunidad académica y administrativa de las instituciones educativas. Por tal razón, se han emprendido programas y proyectos en este sentido. El Gobierno ha hecho millonarias inversiones en la compra de tecnología de punta, con miras a capacitar directivos y docentes en el uso y apropiación de las TIC.

Con referencia a lo anterior, se pueden nombrar, entre otros, el Programa de Formación y Certificación Internacional en Competencias Básicas para el uso de TIC, financiado por el Ministerio de Educación Nacional y ejecutado por la Fundación Certificación Internacional de Competencias Digitales (ICDL) Colombia, y el Programa Nacional en Nuevas Tecnologías, Conexión Total, entre otros. Con todos estos proyectos y lo establecido por el Consejo Nacional de Política Económica y Social (Conpes), se han dado grandes saltos en la incorporación de las TIC en Colombia,

El proceso de apropiación de TIC para el desarrollo profesional docente, plantea dos procesos dinámicos y permanentes de preparación subjetiva (Sensibilización e Inclusión) que ayudan a enfrentar temores, resistencias o dificultades, o bien, que ayudan a fortalecer, desde lo actitudinal, los aprendizajes mediados por la tecnología. Se espera aportar a la formación de un docente innovador en y desde el uso de las TIC para el fortalecimiento de su área o disciplina de desempeño y el desarrollo permanente de competencias en los estudiantes (ICDL, 2014).

Por su parte, Muñoz (2012: 68) concluye que existe escasa o nula utilización de los equipos informáticos en el quehacer docente; no hay apropiación del uso y manejo de las TIC; existen temores hacia el cambio que implica conocer y utilizar las TIC; hay una falta de actitudes y conocimientos en el manejo de las TIC; se ve una carencia de recursos económicos institucionales y personales para capacitación; hay ausencia de programas de capacitación virtual y presencial desde políticas públicas que estén al alcance del docente, y existen deficiencias en el manejo de información tendiente a mejorar las capacidades y habilidades de los docentes, con miras a elevar la calidad de la educación y la calidad de vida de la comunidad educativa.

Finalmente, Valencia y Camargo (2013: 32) concluyen, en primera medida, que el país y sus instituciones educativas necesitan no solo de normas, políticas y acciones de aplicación nacional y local, sino también acciones que permitan robustecer la articulación de gestiones que posibiliten: una mejora en la eficiencia en el uso de recursos públicos dirigidos a los fines mencionados, desde el aprovechamiento de las TIC; y un mayor impacto de las acciones desarrolladas hasta la fecha. Lo anterior permitiría poner fin a la existencia de 'diferentes' países dentro de Colombia, donde algunos territorios cuentan con menos recursos, y las áreas rurales no conocen, por ejemplo, las oportunidades que otorgan las TIC en el desarrollo de las comunidades, desde sus instituciones educativas.

Sumado a lo anterior, los aportes teóricos alrededor de las TIC y su incorporación a los procesos de enseñanza-aprendizaje han venido a fortalecer aún más este amplio terreno de estudio. En tal sentido, Cabero (1989: 5), quien delimita el concepto de TIC, reconoce que para una caracterización de las TIC, estas "giran en torno a cuatro medios básicos: la informática, la microelectrónica, los multimedia y las comunicaciones".

Otro aporte significativo lo realiza Marqués (2002: 2), quien presenta las competencias que se deben tener frente a las TIC en el marco del proceso educativo, las cuales se pueden resumir así: actitud positiva hacia las TIC, instrumento de nuestra cultura que conviene saber utilizar y aplicar en muchas actividades domésticas y laborales, usos de las TIC en el ámbito educativo, uso de las TIC en el campo de su área de 
conocimiento, destreza las TIC en sus actividades, hábito de planificar el currículum integrando las TIC, proponer actividades formativas a los alumnos que consideren el uso de TIC y evaluar el uso de las TIC en la comunidad académica.

Por consiguiente, la apropiación de las TIC, sus usos y aplicaciones en el marco de las instituciones educativas del sector oficial del municipio de Sincelejo está condicionada por el conocimiento, la operacionalización y actitud de la comunidad académica frente a los recursos e infraestructura tecnológica disponible, toda vez que los nuevos ambientes educativos así lo requieren, aprovechando de forma efectiva la infraestructura tecnológica disponible con el concurso de docentes y estudiantes desde su uso dentro y fuera de las aulas de clase.

\section{Metodología}

Con el fin de estudiar las categorías señaladas por la investigación, se planteó un enfoque mixto, con el que se usan herramientas cualitativas y cuantitativas en la mayoría de sus etapas Es conveniente su combinación para obtener información que permita ser triangulada. Con esta alternativa se busca tener la posibilidad de encontrar diferentes caminos para contar con una comprensión e interpretación lo más amplia del fenómeno en estudio (Ruiz y Borboa, 2013).

Por otra parte, se utilizó un tipo de investigación consistente en la descripción exacta de las actividades, recursos, procesos, docentes y estudiantes. Su meta no se limita a la recolección de datos, sino a la predicción e identificación de las relaciones que existen entre dos o más categorías o variables. Aquí se abordó el interrogante acerca del nivel de conocimiento, la aplicación y uso de las TIC por parte de los docentes, estudiantes y directivos de las instituciones educativas del sector oficial del municipio de Sincelejo.

Para ello se focalizaron los grados 10 y 11 de las 35 instituciones del sector, distribuidos de la siguiente manera: 7.526 estudiantes matriculados, 334 docentes adscritos a los mismos grados y 35 rectores de las instituciones educativas del municipio. La escogencia de la muestra se hizo por medio de un muestreo aleatorio probabilístico simple, con un nivel de confianza del $95 \%$, lo que sugiere un número de 179 para un $53.5 \%$ del total de los docentes, 508 para un $14.81 \%$ del total de los estudiantes y 32 directivos para el $91.4 \%$ Estos últimos fueron escogidos con el criterio de inclusión no aleatorio y por su disponibilidad.

Con el mismo propósito se utilizaron como técnicas de recolección de datos la encuesta y la entrevista, dirigida a docentes y a estudiantes, y a directivos, respectivamente. Para ello fue necesario elaborar y aplicar un cuestionario de once preguntas cerradas a docentes $y$ estudiantes, y una entrevista estructurada a directivos, siendo validados dichos instrumentos con una prueba piloto para el cuestionario y una valoración por expertos para la entrevista estructurada.

Paso seguido, el trabajo de campo consistió en la aplicación de los cuestionarios de las encuestas a docentes y estudiantes, y las entrevistas estructuradas en las treinta y cinco instituciones del municipio de Sincelejo, con el apoyo y consentimiento informado de la Secretaría de Educación Municipal, con un total de quince cuestionarios por institución a estudiantes de los grados 10 y 11 (aproximados a 508 cuestionarios), seis (aproximados a 179 cuestionarios) aplicados a docentes por institución, y una entrevista a cada rector de las instituciones visitadas (tres de los cuales no se encontraron disponibles).

\section{Resultados y discusión}

Los resultados del estudio son la descripción de las categorías en relación con los razonamientos involucrados en el uso y apropiación de las TIC por parte de docentes, estudiantes y directivas de las instituciones educativas del sector oficial del municipio de Sincelejo, organizando los 
hallazgos de acuerdo con tres categorías: conocimientos referentes a las TIC, usos de las TIC por parte de la comunidad académica e Infraestructura tecnológica y currículo.

\section{Conocimientos referentes a las TIC}

El $90 \%$ de los estudiantes y el $100 \%$ de los docentes y directivas afirman entender el término $\mathrm{TIC}$, lo relacionan directamente con la tecnología, equipos y herramientas que potencializan el proceso de enseñanza aprendizaje.

Dentro de la misma categoría, $17 \%$ de los estudiantes afirma que las TIC hacen todo el tiempo parte de su cotidianidad, mientras que el $71 \%$ de los docentes lo afirman. Esto muestra una marcada diferencia de percepción de las TIC entre estudiantes y docentes (figuras 1 y 2 ).

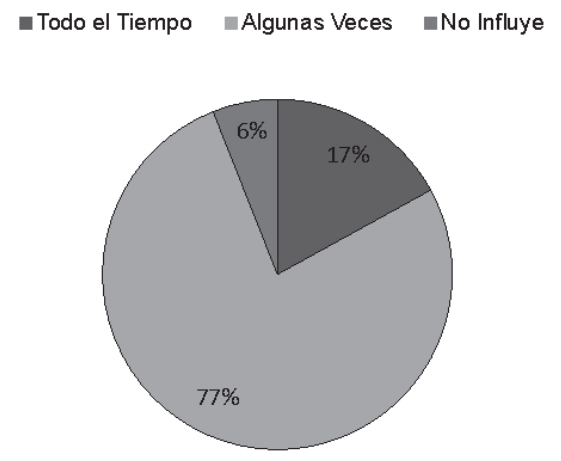

Figura 1: Consideraciones acerca de la TIC los Estudiantes, en su quehacer cotidiano

-Todo el Tiempo $\quad$ Algunas Veces

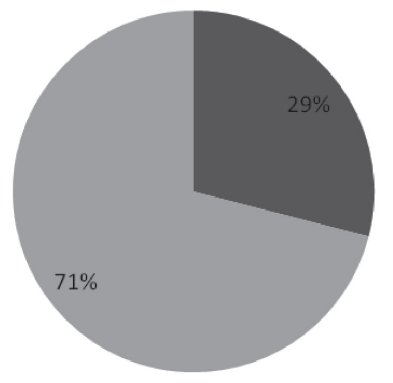

Figura 2: Consideraciones acerca de la TIC en los docentes, en su quehacer cotidiano

\section{Usos de las TIC por parte de la comunidad académica}

El 66\% de los estudiantes encuestados afirma que sus docentes no utilizan las TIC en el desarrollo

-Si No No responde

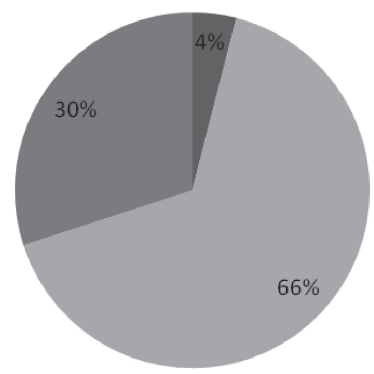

Figura 3. Uso de las TIC en el aula de clases por parte de los profesores

En contraste, el $83 \%$ de los docentes afirma que con frecuencia utilizan las TIC en los procesos de enseñanza-aprendizaje, como lo indica la Figura 4.

$\square \mathrm{Si} \sim$ No

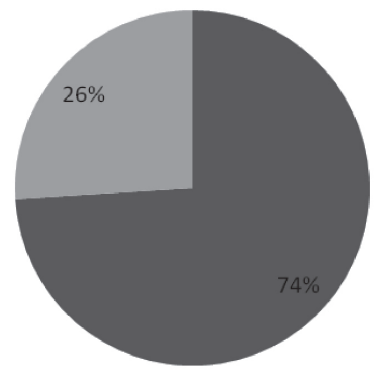

Figura 4: Frecuencia del uso de las TIC por parte de los profesores

Por otra parte, los estudiantes reconocen con un $74 \%$ que sus docentes tienen un manejo adecuado de las TIC en las clases (Figura 5).

Los docentes a su vez, afirman, con un $71 \%$ que el uso de las TIC ha ayudado a los estudiantes a potencializar sus capacidades en el desarrollo de las asignaturas. Así lo indica la Figura 6.

Estos mismos docentes, al interrogárseles por la 


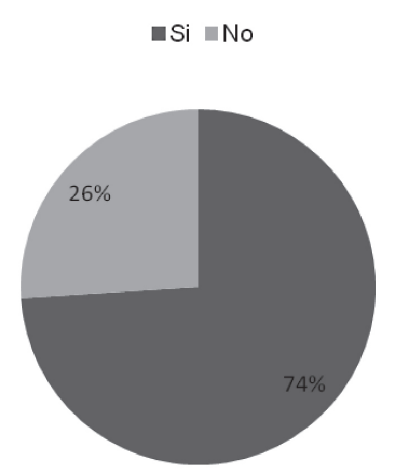

Figura 5: Manejo de las TIC por parte de los profesores

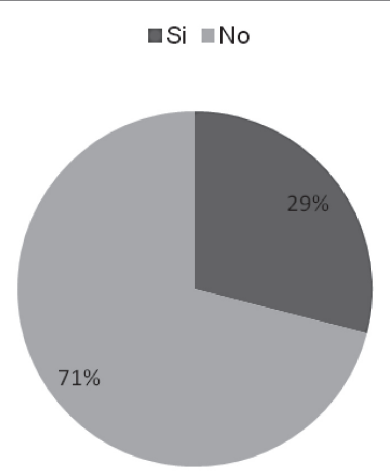

Figura 6: La relevancia del uso de las TIC en el desarrollo de las clases.

frecuencia con la cual usan las TIC en el proceso de enseñanza-aprendizaje, responden con un $83 \%$ que algunas veces, tal como se ilustra en la Figura 7.

\section{Infraestructura tecnológica y currículo}

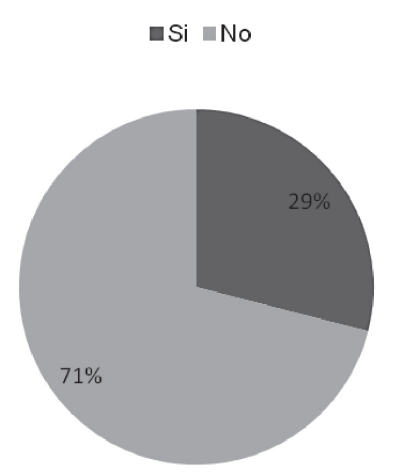

Figura 7: Frecuencia en el Uso de la TIC por parte de los profesores.
La categoría tres se apoyó fundamentalmente en los directivos docentes de cada una de las instituciones educativas, enfocada a la infraestructura educativa y recursos disponibles por la institución. El $100 \%$ de las instituciones del municipio cuenta con una sala de sistemas dotada con accesibilidad y conectividad, de acuerdo con lo expresado por los directivos. En la Figura 8 se detalla el tipo de recursos con que cuentan las instituciones educativas del sector oficial del municipio de Sincelejo.

Como se puede apreciar en la Figura 8, las instituciones educativas estudiadas cuentan en primer lugar con portátiles, seguido de amplificaciones, videoproyectores (video-beam) e impresoras.

Ahora bien, de acuerdo con las categorías presentadas y los hallazgos encontrados por la aplicación de los instrumentos, se pueden trazar tres líneas gruesas a manera de resultados. Una primera línea, referente a los conocimientos: docentes, estudiantes y directivas tienen conocimientos diversos acerca de las TIC, y aunque identifican su significado y lo asocian de forma adecuada a las herramientas tecnológicas que median el proceso de formación, este conocimiento no pasa de ser nocional; es decir, se constituye como mera noción del objeto (TIC), sin que aquello signifique el conocimiento de su uso en el proceso de enseñanza-aprendizaje. Esta evidencia contraría lo que plantea la Unesco desde el año 2008 en cuanto a la plenitud de conocimientos para el manejo de las TIC y su aplicación por parte de docentes y estudiantes.

En otros términos, docentes y estudiantes no son competentes desde lo cognitivo y operacional frente a las TIC, su uso y apropiación en el marco de los procesos de enseñanzaaprendizaje. Como lo señala Marqués (2002) las competencias necesarias para apropiar las TIC son: una actitud positiva frente a estas, un uso pertinente y frecuente en los ambientes de aprendizaje, una cultura mediada por las TIC y un currículo permeado por las nuevas herramientas tecnológicas. Una segunda línea está dada por el uso en sí de las TIC en el aula y fuera de ella. 


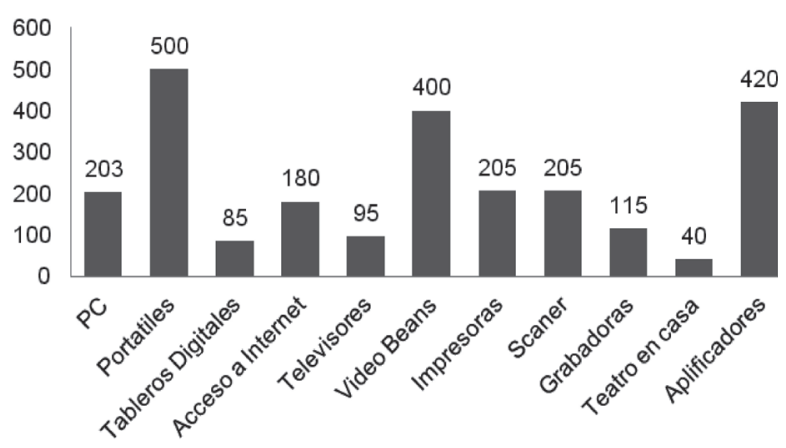

Figura 8: Distribución de recursos existentes en las I.E.

En este punto, estudiantes y directivas afirman que el uso de los docentes de las herramientas tecnológicas es deficiente y, a su vez, los docentes manifiestan solo utilizar una minoría de estas herramientas, aunque frecuentemente.

Lo que reflejan los resultados obtenidos es una subutilización general de muchos de los recursos tecnológicos con que se dotan a las instituciones del sector oficial, bien sea por falta de manejo técnico, falta de planeación en clases o simplemente por una cultura subyacente de resistencia al cambio frente a las TIC. Así, equipos con múltiples usos y aplicaciones son tomados solo a partir de una o dos funciones, o dejados en desuso en espacios como bibliotecas o salas de audiovisuales. Muñoz (2012) concluye que existe escasa o nula utilización de los equipos informáticos en el quehacer docente, no hay apropiación del uso y manejo de las TIC y existen temores hacia el cambio que implica conocer y utilizar las TIC.

Y en una tercera línea de análisis se encuentra la gestión curricular de las TIC por parte de las directivas de las instituciones, que asumen la responsabilidad de una gestión del cambio que modernice la educación hacia la construcción de ciudadanos más competitivos. Esto ha llevado a jornadas de capacitación en TIC y áreas afines, adquisición de infraestructura tecnológica, cambio de metodología hacia didácticas más relacionadas con las $\mathrm{TIC}$, entre otras estrategias que, sin la base de la reflexión acerca del impacto de las TIC en la comunidad académica y su efectividad como herramientas disponibles al estudiante y docente, no generan el impacto esperado en la comunidad académica. Esto coincide con el estudio desarrollado por Muñoz (2012), quien reconoce que no basta una infraestructura suficiente para construir una cultura de las TIC y su apropiación en las instituciones educativas.

Así las cosas, los vectores causa-efecto acerca de las TIC, su apropiación y uso no radican exclusivamente en la existencia de equipos disponibles, ni salas acondicionadas, ni arduas jornadas de capacitación a los docentes; el problema tiene una de sus principales fuentes en la cultura organizacional, como imaginario colectivo formal o informal que caracteriza a un grupo humano. Esta cultura es el ambiente sobre el cual hay que dirigir esfuerzos para un cambio radical en la manera, forma y razón de educar.

Esto se puede observar a la luz de los anteriores resultados, donde docentes, directivos y estudiantes no estiman que haya un uso uniforme y una apropiación de las TIC en el marco de las instituciones educativas del sector oficial del municipio de Sincelejo, lo que se manifiesta en la subutilización de una infraestructura tecnológica, que desaprovecha una inversión significativa hecha por el Estado.

\section{Conclusiones y recomendaciones}

Los nuevos ambientes de aprendizaje suponen retos a quienes los usan $y$, a su vez, nuevas estructuras y herramientas para responder a nuevos propósitos y filosofías curriculares. Esta dinámica incluye a las TIC como herramienta de un proceso de enseñanza-aprendizaje cambiante, al tiempo que exige a docentes, estudiantes y directivas una apropiación y uso esperados para el logro de sus objetivos. Con base a este enfoque y como fruto de los hallazgos analizados, se presentan las siguientes conclusiones:

El nivel de conocimiento de los docentes, estudiantes y directivas de las instituciones 
educativas del sector oficial del municipio de Sincelejo es básico, a pesar de haber sido capacitados en manejo de las TIC e interactuar con estas cotidianamente. Se continúa nocionalmente asociando a las TIC solo con la identificación de cada recurso tecnológico existente en la institución, sin haber una conciencia de su aplicación e impacto en el proceso de formación.

El uso de las TIC se restringe a consultas, correos electrónicos y a las redes sociales, siendo la categoría de uso más característica de las TIC en el ámbito de las instituciones educativas, puesto que no se hace practico un nivel de uso y apropiación mediado por la tecnología en los ambientes educativos. No existe entre estudiantes y docentes un consenso en cuanto a la aplicación de las TIC en el aula.

Los intereses por lo nuevo o por explicar lo desconocido son relativamente bajos por parte de los docentes. La resistencia al cambio y la acomodación a una zona de seguridad laboral generan un ambiente poco propicio para la implementación de las TIC en el proceso de enseñanza-aprendizaje en las instituciones educativas del sector oficial del municipio de Sincelejo.

La ausencia de una cultura organizacional orientada al cambio, la innovación y el uso de las TIC como herramientas pedagógicas de un nuevo enfoque curricular, conforman la raíz de la problemática planteada. Los niveles de uso y apropiación están relacionados con dicha cultura dentro de las instituciones educativas.

Así mismo, la operacionalización de las conclusiones orientada a una acción sostenible permitió destacar las siguientes recomendaciones:

(a) Desde las directivas de la institución adelantar una estrategia de gestión del cambio, considerando de manera integral todos los aspectos constituyentes de la comunidad académica y minimizando la resistencia al cambio, (b) Redefinir el modelo pedagógico con una visión tecnológica práctica, centrado en las necesidades y exigencias propias del entorno del siglo XXI, (c) Monitorear el uso de las TIC como herramienta pedagógica dentro y fuera del aula, retroalimentando los procesos de implementación y acompañando a docentes y estudiantes.

\section{Referencias}

Cabero, J. (1989). Tecnología educativa: utilización didáctica del vídeo. PPU, Barcelona.

Fundación Certificación Internacional de Competencias Digitales Colombia (2014). Certificación Internacional de Competencias Digitales, Recuperado de: http://www.icdlcolombia.com/sitio/ noticias/20-icdl-colombia-noticias-/98men.html.

Marqués, P. (2002). "Las TIC y sus aportaciones a la sociedad" [en línea], recuperado de: http://dewey.uab.es/pmarques/tic.htm

Ministerio de Educación Nacional (2011). Enlaces tecnológicos. Gobierno de Chile, recuperado de: http://www.enlaces.cl/ index.php? $\mathrm{t}=44 \& \mathrm{i}=2 \& \mathrm{cc}=1614 \& \mathrm{tm}=2$.

Muñoz, L. (2012). "Apropiación, uso y aplicación de las TIC en los procesos pedagógicos que dirigen los docentes de la institución educativa núcleo escolar rural Corinto", Universidad Nacional de Colombia Sede Palmira, Maestría en Enseñanza de las Ciencias Exactas y Naturales, Facultad de Ingeniería y Administración, Palmira.

Organización de las Naciones Unidas para la Educación, la Ciencia y la Cultura (2008 [2002]). A Curriculum for Schools and Programme of Teacher Development; Division of Higher Education, Unesco, París, p. 3, recuperado de http://www. 
unescobkk.org/index.php?id=3127

Ruiz, M.; Borboa, M. (2013). “El enfoque mixto de investigación en los estudios fiscales", Revista Académica de Investigación, núm. 13, agosto.

Valencia, J.; Camargo, K. (2013). “Estrategias para el fortalecimiento de las TIC en las escuelas en Colombia", Universidad del Norte, resuperado de: http:// www.colombiadigital.net/nuestraspublicaciones/educacion/item/5581estrategias-para-el-fortalecimiento-delas-tic-en-las-escuelas-en-colombia.html 
